\title{
Challenging management of prosthetic valve infective endocarditis: usefulness of 18F-FDG PET/CT in diagnosis and follow up
}

L. Romani ${ }^{1}$, M. De Luca ${ }^{1}$, L. Gargiullo' ${ }^{1}$, P. Zangari ${ }^{1}$, G. Rinelli ${ }^{2}$, M. Chinali ${ }^{2}$, F.I. Calo' Carducci ${ }^{1}$, P. D'Argenio ${ }^{1}$

${ }^{1}$ Bambino Gesù Children's Hospital-IRCCS, Immunological and Infectious Disease Unit- University Department of Pediatrics, Rome, Italy.

2Bambino Gesu Children's Hospital-IRCCS, Pediatric Cardiology and Arhythmology Unit, Rome, Italy

\section{Background}

The use of foreign material to correct Congenital Heart Diseases (CHD) have contributed to increase the incidence of prosthetic valve infective endocarditis (PVE). The diagnosis of PVE is challenging, symptoms can be atypical and blood cultures are often negative. Modified Duke Criteria and echocardiography are mainly used for the diagnosis of PVE. 18F-FDG PET/CT might be a supplementary diagnostic technique.

\section{Case Presentation}

A 14-year-old girl with surgically corrected transposition of great arteries was admitted to our hospital for fever of unknown origin (FUO) and elevated inflammatory markers.
She had received a prosthetic aortic valve and pulmonary homograft replacement eight months before. Echocardiography resulted negative, as well as blood cultures. Considering the high clinical suspicion of PVE, an 18F-FDG PET/TC was performed showing aortic uptake and evidence of splenic embolism. The 18F-FDG PET/CT performed after 6 weeks of antibiotic therapy showed a signal reduction on the prosthetic valve. A 19-year-old boy, with a biological aortic valve implanted 5 years before, was admitted to our hospital for persistent fever. Blood tests showed normal WBC count and slightly elevated CRP, with negative blood culture.
Serology

for

Coxiella

burnetii resulted diagnostic for chronic infection. Echocardiography resulted negative; 18F-FDG PET/CT was performed showing aortic PVE then specific antibiotic therapy was started.

\section{Discussion}

Early diagnosis is critical since delay in therapy has been associated with a poor outcome. Our cases support the usefulness of $18 \mathrm{~F}$ FDG $P E T / C T$ in the suspicion of endocarditis with negative echocardiography. Further studies are necessary to determine if the repetition of the 18F-FGD PET/CT during follow up is useful to monitor the response to antibiotic therapy and the correct timing. 